\title{
ON A CONJECTURE OF ATKIN FOR THE PRIMES 13, 17, 19, AND 23
}

\author{
P. Guerzhoy
}

\begin{abstract}
In his paper [2], Atkin pioneered computer investigations of divisibility properties of Fourier coefficients of the modular invariant by powers of $13,17,19$, and 23 . On the basis of these computations he formulated certain conjectures in $[2,3]$. In particular, the question why similar congruence properties occur for these primes is posed in [2]. We show how a combination of Serre's theory of $p$-adic modular forms and Hida's Control Theorem explains the phenomenon.
\end{abstract}

\section{Introduction}

For a rational prime $p$ the action of $U_{p}=U$-operator on formal power series in variable $q$ is defined by $f \mapsto f \mid U$, where

$$
f=\sum a(n) q^{n} \quad \text { and } \quad f \mid U=\sum a(p n) q^{n} .
$$

In his papers $[2,3]$ Atkin formulated conjectures about certain divisibility properties of the $q$-expansion coefficients of the modular invariant $j$. Roughly, it turns out that the more one applies the $U$-operator to $j$, the closer $p$-adically it becomes to a Hecke eigenform. This takes place for primes $p \leq 23$ and was discovered by Atkin on the basis of extensive numerical experiments. As far as the current author knows, after this research the $U$-operator, which was well-known well before, acquired its personal name "Atkin's $U$-operator".

Atkin summarizes one of his conjectures in [3]:

"Given "any" entire function on $\Gamma_{0}(p)$, then repeated application of $U$ leads to a series of Fourier coefficients which become multiplicative modulo increasing powers of $p . '$

We use the common notation $q=e^{2 \pi i \tau}$ with $\tau$ in the complex upper half-plane. Let $\mathcal{F}$ be a meromorphic modular function (of weight 0 ) on $\Gamma_{0}\left(p^{N}\right)$ for a prime $p$ and $N \geq 0$. Denote by $c(\mathcal{F}, n)$ its $q$-expansion coefficients:

$$
\mathcal{F}=\sum_{n \gg-\infty} c(\mathcal{F}, n) q^{n}
$$

and assume that $c(\mathcal{F}, n) \in \mathbb{Q}$. Let $t_{m}(\mathcal{F}, n)=c\left(\mathcal{F}, p^{m} n\right) / c\left(\mathcal{F}, p^{m}\right)$ for a positive integer $m$. For a function on integers it is assumed that the value is zero if the argument is not an integer.

With these notations we formulate the above claim.

Received by the editors June 18, 2005.

2000 Mathematics Subject Classification. Primary 11F33.

Supported by NSF grant DMS-0501225. 
Conjecture (Atkin). For any positive integer $\alpha$ there exists a positive integer $M=$ $M(\alpha)$ such that the congruences

$$
t_{m}(\mathcal{F}, n l)-t_{m}(\mathcal{F}, n) t_{m}(\mathcal{F}, l)+l^{-1} t_{m}(\mathcal{F}, n / l) \equiv 0 \bmod p^{\alpha}
$$

and

$$
t_{m}(\mathcal{F}, n p)-t_{m}(\mathcal{F}, n) t_{m}(\mathcal{F}, p) \equiv 0 \bmod p^{\alpha} .
$$

hold for every prime $l \neq p$, every positive integer $n$ and every integer $m \geq M$.

In the case when

$$
\mathcal{F}=j=q^{-1}+744+196884 q+\ldots=q^{-1}+744+\sum_{n \geq 1} c(n) q^{n}
$$

is the usual elliptic modular function on $S L(2, \mathbb{Z})$, the congruences claimed in the conjecture were discovered on the basis of numerical experiments [2]. Conjecturally in this case one can put $M=\alpha$. Later, there appeared a proof for the case $p=13$ (with $M=\alpha$ ) by Koike [10] as an application of a profound result of Koike and a result of Atkin and O'Brien [4]; the latter is specific for the case $p=13$. Another proof for the case $p=13$ which makes use of a result of Dwork [6] was presented by Katz [9] in the framework of his theory of $p$-adic modular forms. A proof for $p=2$ (with a more precise $M<[\alpha / 4]$ ) was found later by Akiyama [1].

The conjecture was tested numerically in [2] exactly for the primes $p=13,17,19$, and 23. In that paper Atkin wrote:

'... it is remarkable that all these primes should exhibit the same behaviour with regard to our multiplicative congruence properties. It thus seems possible that some entirely different method or theory may exist which would give an uniform proof of all these cases.'

We present such a uniform approach in this paper and prove the conjecture for a certain class of meromorphic modular functions which contains $j$ along with infinitely many other functions, in particular, polynomials in $j$. In the cases when $p=13,17,19$, or 23, we have $\operatorname{dim} S_{p-1}\left(S L_{2}(\mathbb{Z}), \mathbb{C}\right)=1$ and the unique normalized cusp form $F_{p-1} \in$ $S_{p-1}\left(S L_{2}(\mathbb{Z}), \mathbb{C}\right)$ is $p$-ordinary (see below for the definitions). In the framework of our approach, this is the key property shared by the primes under consideration.

We formulate our results in Section 2 and prove them in Section 3. The essential ingredients of the proof are (a version of) Hida's Control Theorem [7, Chapter 7] and Serre's theory of $p$-adic modular forms $[12,13]$.

Throughout this paper $p$ denotes one of the primes $13,17,19$, or 23 .

\section{Statement of results}

Fix an algebraic closure $\overline{\mathbb{Q}}_{p}$ of the $p$-adic field $\mathbb{Q}_{p}$ and an embedding of $\overline{\mathbb{Q}}$ into $\overline{\mathbb{Q}}_{p}$. Thus any algebraic number can be regarded as a $p$-adic number. We denote by $v_{p}(x)$ the $p$-adic ordinal of an algebraic number $x$ (with respect to the fixed embedding). For a formal power series $h=\sum u(n) q^{n} \in \overline{\mathbb{Q}}[[q]]$ define $v_{p}(h)=\inf _{n} v_{p}(u(n))$, and say $h \equiv g \bmod p^{\beta}$ if $v_{p}(h-g) \geq \beta$.

For an even positive integer $k$, an integer $N \geq 0$ and a $\mathbb{Z}$-algebra $A$ inside $\mathbb{C}$ we denote by $S_{k}\left(\Gamma_{0}\left(p^{N}\right), A\right)$ the $A$-module of cusp forms of weight $k$ with respect to the congruence subgroup $\Gamma_{0}\left(p^{N}\right)$ such that all their $q$-expansion coefficients belong to $A$. 
We assume $\Gamma_{0}(1)=S L(2, \mathbb{Z})$. It is known that $S_{k}\left(\Gamma_{0}\left(p^{N}\right), A\right)=S_{k}\left(\Gamma_{0}\left(p^{N}\right), \mathbb{Z}\right) \otimes A$ for $A \subset \mathbb{C}$, and we set $S_{k}\left(\Gamma_{0}\left(p^{N}\right), A\right)=S_{k}\left(\Gamma_{0}\left(p^{N}\right), \mathbb{Z}\right) \otimes A$ for a $\mathbb{Z}$-algebra $A$ inside $\overline{\mathbb{Q}}_{p}$.

Recall that a Hecke operator $T_{l}$ for a rational prime $l$ with $\left(l, p^{N}\right)=1$ is defined by $f \mapsto f \mid T_{l}$, where

$$
f=\sum_{n>0} a(n) q^{n} \quad \text { and } \quad f \mid T_{l}=\sum_{n>0}\left(a(l n)+l^{k-1} a(n / l)\right) q^{n} .
$$

The operator $T_{l}$ acts on $S_{k}\left(\Gamma_{0}\left(p^{N}\right), A\right)$. We also put $T_{p}=U$ if $N \geq 1$. A modular form $f \in S_{k}\left(\Gamma_{0}\left(p^{N}\right), A\right)$ is a simultaneous Hecke eigenform if $f \mid T_{l}=\lambda_{l} f$ for some $\lambda_{l} \in A$ for all $l$. In this case $a(1) \neq 0$ and $\lambda_{l}=a(l) / a(1)$. It is known that all $\lambda_{l}$ are algebraic integers, in particular, $v_{p}\left(\lambda_{l}\right) \geq 0$. It is also known that for any Hecke eigenform the extension of $\mathbb{Q}$ generated by $\lambda_{l}$ for all $l$ is finite. Every linear space $S_{k}\left(\Gamma_{0}\left(p^{N}\right), \mathbb{C}\right)$ is finite-dimensional and has a basis which consists of simultaneous Hecke eigenforms which have their Fourier expansion coefficients in $\overline{\mathbb{Q}}$.

Let $\mathcal{F}$ be a meromorphic modular function on $\Gamma_{0}\left(p^{N}\right)$ for an integer $N \geq 0$ with the $q$-expansion (1). In order to formulate our result we have to replace the word "any" in Atkin's conjecture by certain conditions on $\mathcal{F}$. In the case when $\mathcal{F}=j$, the fact that $(j-744) \mid U$ is a $p$-adic cusp form of weight 0 was first observed by Deligne [12, Remarque 3.2]. We need a similar statement for our $\mathcal{F}$ (see Proposition 1 below). For this, we allow $\mathcal{F}$ to have poles at cusps (including infinity) and we impose a certain restriction which pertains to its poles in the interior of the complex upper half-plane.

If $N>0$ we require that $\mathcal{F}$ is holomorphic in the interior of the complex upper halfplane. Otherwise (i.e. if $\mathcal{F}$ is a modular function on $S L(2, \mathbb{Z})$ ) choose a fundamental domain of the action of $S L(2, \mathbb{Z})$, and let the poles be at points $\tau_{i}$ in the fundamental domain. Consider the (finite) product

$$
\mathcal{D}(\mathcal{F}, X)=\prod_{i}\left(X-j\left(\tau_{i}\right)\right)
$$

We require this polynomial to have rational $p$-integral coefficients:

$$
\mathcal{D}(\mathcal{F}, X) \in \mathbb{Q}[X] \cap \mathbb{Z}_{p}[X]
$$

Denote by $\overline{\mathcal{D}}(\mathcal{F}, X) \in \mathbb{F}_{p}[X]$ the polynomials obtained by reducing the coefficients modulo $p$. Let $S_{p}(X) \in \mathbb{F}_{p}[X]$ be the supersingular polynomial at $p$, namely

$$
S_{p}(X)=\left\{\begin{array}{ccc}
X+8 & \text { if } & p=13 \\
X(X+9) & \text { if } & p=17 \\
(X+1)(X+12) & \text { if } & p=19 \\
X(X+4)(X+20) & \text { if } & p=23
\end{array}\right.
$$

We require $\overline{\mathcal{D}}(\mathcal{F}, X)$ to divide a power of $S_{p}(X)$ :

$$
S_{p}(X)^{t} / \overline{\mathcal{D}}(\mathcal{F}, X) \in \mathbb{F}_{p}[X]
$$

for an integer $t \geq 0$.

Theorem 1. Let $\mathcal{F}$ be a meromorphic modular function with respect to $\Gamma_{0}\left(p^{N}\right)$ for some $N \geq 0$ with the q-expansion (1) with rational coefficients $c(\mathcal{F}, n) \in \mathbb{Q}$. Assume that $v_{p}\left(c\left(\mathcal{F}, p^{n}\right)\right)$ are bounded. 
If $N>0$ we assume that $\mathcal{F}$ is holomorphic in the interior of the complex upper half-plane.

If $N=0$ we assume that the locus of those poles of $\mathcal{F}$ which lie in the interior of the upper half-plane satisfies (3) and (4).

Then for any positive integer $\alpha$ there exists $M=M(\alpha)$ such that for every $m \geq M$ there exists a simultaneous Hecke eigenform $f \in S_{k}\left(\Gamma_{0}(p), \mathbb{Z}_{p}\right.$ ) (for an even positive integer $k$ ) such that

$$
f \equiv(\mathcal{F}-c(\mathcal{F}, 0)) \mid U^{m} \bmod p^{\alpha} .
$$

Remark. The conclusion of Theorem 1 remains true and in fact becomes vacuous without the assumption that $v_{p}\left(c\left(\mathcal{F}, p^{n}\right)\right)$ are bounded. We add the assumption because we need it for the applications.

If a modular function $\mathcal{F}$ satisfies the conditions of Theorem 1 then Atkin's conjecture holds for $\mathcal{F}$. This follows at once from Theorem 1, the definition of Hecke operators $(2)$ and the assumption that $v_{p}\left(c\left(\mathcal{F}, p^{n}\right)\right)$ are bounded.

Corollary 1. The function $\mathcal{F}=j$ satisfies the conditions of Theorem 1 and, therefore, Atkin's conjecture is true for $\mathcal{F}=j$.

Proof. All we need to check is the boundedness of $v_{p}\left(c\left(p^{n}\right)\right)$. We claim that in fact $v_{p}\left(c\left(p^{n}\right)\right)=0$ for all $n \geq 0$. The space $S_{p-1}\left(S L_{2}(\mathbb{Z}), \mathbb{C}\right)$ is one-dimensional. Denote by $F_{p-1}=\sum_{n>0} b(n) q^{n}$ the unique cusp form (which is therefore a Hecke eigenform) in this space normalized such that $b(1)=1$. Then $b(n)$ are known to be rational integers and the congruences

$$
c(p) F_{p-1} \equiv(j-744) \mid U \bmod p
$$

with $c(p) \not \equiv 0 \bmod p$ are proved by Serre in $[13,6.16]$ (this congruence for $p=13$ was proved earlier by Newman [11]). Since $F_{p-1}$ is a normalized eigenform for $T_{p}$, it follows from (2) that $b(p) b\left(p^{n-1}\right)=b\left(p^{n}\right)+p^{p-2} b\left(p^{n-2}\right)$, and an induction argument on $n$ implies that $b\left(p^{n}\right) \not \equiv 0 \bmod p$ for $n \geq 0$. For the induction base we calculate the quantities $b(p)$

$$
\begin{array}{ccccc}
p & 13 & 17 & 19 & 23 \\
b(p) & -577738 & 1646527986 & 1487499860 & -73845437470344
\end{array}
$$

in order to check that

$$
b(p) \not \equiv 0 \bmod p .
$$

Now, by $(5), c(p) b\left(p^{n-1}\right) \equiv c\left(p^{n}\right) \bmod p$, and this implies our claim, namely that

$$
c\left(p^{n}\right) \not \equiv 0 \bmod p \quad \text { for any } \quad n \geq 0 \text {. }
$$

There are many functions $\mathcal{F}$ which satisfy the conditions of Theorem 1 . In particular, we prove the following.

Theorem 2. Let $P \in X \mathbb{Z}[X]$. Consider the modular function $\mathcal{F}=P(j)+\beta j+\gamma$ with $\beta, \gamma \in \mathbb{Z}$. There is a modulo $p$ residue class $\xi(P)$ such that the conditions of Theorem 1 are satisfied and therefore the congruences claimed in Atkin's conjecture hold for $\mathcal{F}$ if $\alpha \not \equiv \xi(P) \bmod p$. 


\section{Proofs of Theorem 1 and 2}

We use Serre's definition of $p$-adic modular forms [12]. Recall that a power series $\phi \in \mathbb{Z}_{p}[[q]]$ is a $p$-adic cusp form of weight 0 if for every integer $\alpha>0$ there exists $\Phi \in S_{k}(S L(2, \mathbb{Z}), \mathbb{Z})$ such that $\Phi \equiv \phi \bmod p^{\alpha}$. Here $k$ is an even positive integer, and by [12, Théorème 1] $k \equiv 0 \bmod (p-1) p^{\alpha-1}$. We consider here only $p$-adic modular forms of weight 0 without constant terms. This allows us to consider cusp forms instead of merely modular forms.

Proposition 1. Let $\mathcal{F}$ be a meromorphic modular function with the q-expansion (1) with rational coefficients $c(\mathcal{F}, n) \in \mathbb{Q}$. Assume that either $\mathcal{F}$ is holomorphic in the interior of the upper half-plane or $N=0$ and the locus of those poles of $\mathcal{F}$ which lie in the interior of the upper half-plane satisfies (3) and (4).

Then $(\mathcal{F}-c(\mathcal{F}, 0)) \mid U^{r}$ is a p-adic cusp form of weight 0 for all sufficiently large $r$.

Proof. Let $\alpha \geq 1$ be a positive integer. We first consider the poles in the interior of the upper half-plane and use a slight variation of the argument of Bruinier and Ono [5, Proof of Theorem 2]. Let $E_{p-1}$ be the Eisenstein series of weight $p-1$ :

$$
E_{p-1}=1-\frac{2 p-2}{B_{p-1}} \sum_{n \geq 1} \sigma_{p-2}(n) q^{n},
$$

and let $Q_{1}, \ldots, Q_{s}$ be its zeros in the fundamental domain of the action of $S L_{2}(\mathbb{Z})$. (Note that $s \leq 3$ since $p \leq 23$.) Let

$$
V_{p}(X)=\prod_{i=1}^{s}\left(X-j\left(Q_{i}\right)\right) .
$$

Then (see, for example, $\left[8\right.$, Theorem 1]) $V_{p}(X) \equiv S_{p}(X) \bmod p$.

Let $Z(X)$ be any lift to $\mathbb{Z}[X]$ of $S_{p}^{t}(X) / \overline{\mathcal{D}}(\mathcal{F}, X) \in \mathbb{F}_{p}[X]$. Since $E_{p-1} \equiv 1 \bmod p$, we conclude that

$$
\mathcal{G}:=E_{p-1}^{t} \frac{\mathcal{D}(\mathcal{F}, j)}{V_{p}^{t}(j)} Z(j) \equiv 1 \bmod p .
$$

Thus

$$
\mathcal{F} \mathcal{G}^{p^{\alpha}} \equiv \mathcal{F} \bmod p^{\alpha},
$$

and the product on the left is holomorphic in the interior of the fundamental domain by construction.

Repeated application of the $U$-operator eliminates the pole at infinity. Consider

$$
\Psi:=\left((\mathcal{F}-c(\mathcal{F}, 0)) \mathcal{G}^{p^{\alpha}}\right) \mid U^{r} \equiv\left(\mathcal{F}-c(\mathcal{F}, 0) \mid U^{r} \bmod p^{\alpha} .\right.
$$

If $r$ is big enough, then $\Psi$ has poles neither in the interior of the fundamental domain nor at infinity. A theorem of Serre [13, Théorème 5.4] implies that $\Psi$ is a $p$-adic cusp form; in other words, there exists $\Phi \in S_{k}(S L(2, \mathbb{Z}), \mathbb{Z})$ for some positive integer $k$ such that $\Phi \equiv \Psi \bmod p^{\alpha}$. Thus $\Phi \equiv\left(\mathcal{F}-c(\mathcal{F}, 0) \mid U^{r} \bmod p^{\alpha}\right.$.

Proof of Theorem 1. Let $\alpha$ be a positive integer, and let $\Phi_{r, \alpha} \in S_{k}\left(S L_{2}(\mathbb{Z}), \mathbb{Z}\right) \subset$ $S_{k}\left(\Gamma_{0}(p), \mathbb{Z}\right)$ be a cusp form such that $\Phi_{r, \alpha} \equiv(\mathcal{F}-c(\mathcal{F}, 0)) \mid U^{r} \bmod p^{\alpha}$. The existence of this cusp form of some weight $k$ is guaranteed by Proposition 1. It follows from a theorem of Serre [12, Théorème 1] that $k \equiv 0 \bmod (p-1) p^{\alpha-1}$. Hida's ordinary 
projector $[7,7.2]$ is defined as the $p$-adic limit $\mathcal{E}=\lim _{n \rightarrow \infty} T_{p}^{n !}$. For a Hecke eigenform $\phi \in S_{k}\left(\Gamma_{0}(p), \overline{\mathbb{Q}}\right)$ with $\phi \mid T_{p}=\lambda_{p} \phi$

$$
\phi \mid \mathcal{E}=\left\{\begin{array}{lll}
\phi, & \text { if } & v_{p}\left(\lambda_{p}\right)=0 \\
0, & \text { if } & v_{p}\left(\lambda_{p}\right)>0 .
\end{array}\right.
$$

Recall that a cusp Hecke eigenform $\phi$ is called $p$-ordinary if $v_{p}\left(\lambda_{p}\right)=0$. The $\mathbb{Z}_{p^{-}}$ module $S_{k}^{\text {ord }}\left(\Gamma_{0}(p), \mathbb{Z}_{p}\right)$ is defined as the image of $\mathcal{E}$ acting on $S_{k}\left(\Gamma_{0}(p), \mathbb{Z}_{p}\right)$. Hecke operators act on $S_{k}^{\text {ord }}\left(\Gamma_{0}(p), \mathbb{Z}_{p}\right)$. Hida's Control Theorem [7, Theorem 7.1] implies that $\operatorname{rank}_{\mathbb{Z}_{p}} S_{k}^{\text {ord }}\left(\Gamma_{0}(p), \mathbb{Z}_{p}\right)$ does not depend on $k \geq 2$ if $k$ is constant modulo $p-1$. Moreover, we have the equality $\operatorname{rank} S_{k}^{\text {ord }}\left(\Gamma_{0}(p), \mathbb{Z}_{p}\right)=\operatorname{rank} S_{k}^{\text {ord }}\left(S L_{2}(\mathbb{Z}), \mathbb{Z}_{p}\right)$, which follows from [7, Proposition 7.2.2]. Thus $\operatorname{rank}_{\mathbb{Z}_{p}} S_{k}^{\text {ord }}\left(\Gamma_{0}(p), \mathbb{Z}_{p}\right)=1$, since $\operatorname{dim} S_{p-1}\left(S L_{2}(\mathbb{Z}), \mathbb{C}\right)=1$ and the unique weight $p-1$ cusp Hecke eigenform $F_{p-1} \in$ $S_{p-1}\left(S L_{2}(\mathbb{Z}), \mathbb{Z}\right)$ is $p$-ordinary by $(6)$. The equality $\operatorname{rank}_{\mathbb{Z}_{p}} S_{k}^{\text {ord }}\left(\Gamma_{0}(p), \mathbb{Z}_{p}\right)=1$ implies that every element of $S_{k}^{\text {ord }}\left(\Gamma_{0}(p), \mathbb{Z}_{p}\right)$ is a simultaneous Hecke eigenform; in particular, this applies to $\Phi_{r, \alpha} \mid \mathcal{E}$. We also have $\Phi_{r, \alpha} \mid \mathcal{E} \in S_{k}\left(\Gamma_{0}(p), \mathbb{Z}_{p}\right)$ by construction, and $\Phi_{r, \alpha} \mid \mathcal{E} \neq 0$ because $v_{p}\left(c\left(\mathcal{F}, p^{n}\right)\right)$ are bounded. Thus $\left(\Phi_{r, \alpha} \mid \mathcal{E}\right) \mid U=\lambda\left(\Phi_{r, \alpha} \mid \mathcal{E}\right)$ with $\lambda \in \mathbb{Z}_{p}^{*}$. The finite dimensional linear space $S_{k}\left(\Gamma_{0}(p), \overline{\mathbb{Q}}_{p}\right)$ has a basis which consists of simultaneous Hecke eigenforms. In particular,

$$
\Phi_{r, \alpha}=\mu \Phi_{r, \alpha} \mid \mathcal{E}+G,
$$

where $\mu \in \overline{\mathbb{Q}}_{p}$, and $G$ is a linear combination over $\overline{\mathbb{Q}}_{p}$ of normalized simultaneous Hecke eigenforms which are not $p$-ordinary. Now apply $\mathcal{E}$ to both sides of the above identity and note that $G \mid \mathcal{E}=0$ and $\mathcal{E}^{2}=\mathcal{E}$ to conclude that $\mu=1$. It follows that there exists $J$ such that $v_{p}\left(G \mid U^{i}\right)>\alpha$ if $i>J$. Set now $M=r+J$ and $f=\lambda^{m-r} \Phi_{r, \alpha} \mid \mathcal{E}$. We have

$$
(\mathcal{F}-c(\mathcal{F}, 0))\left|U^{m} \equiv \Phi_{r, \alpha}\right| U^{m-r} \equiv f \bmod p^{\alpha}
$$

for $m>M$ as required.

Proof of Theorem 2. All we need to check is the boundedness of $v_{p}\left(c\left(\mathcal{F}, p^{n}\right)\right)$. It is thus sufficient to show that for a suitable choice of $\beta$

$$
c\left(\mathcal{F}, p^{n}\right) \not \equiv 0 \bmod p \quad \text { for all } \quad n \geq 0 .
$$

Recall from [12] that for a $p$-adic modular form $g$ the filtration $w(g)$ is defined as the minimal weight of a classical modular form $G$ such that $g \equiv G \bmod p$. Let $S=P(j)+\gamma-c(P(j)+\gamma, 0)$ and $J=j-c(j, 0)=j-744$. Proposition 1 implies that both $S \mid U^{r}$ and $J \mid U^{r}$ are $p$-adic cusp forms for sufficiently big $r$. By a result of Serre, [12, Théorème 6(i)], application of $U$ decreases the filtration if the filtration was bigger than $p-1$. Thus there exists $R$ such that $S \mid U^{R} \equiv B F_{p-1} \bmod p$ and $J \mid U^{R} \equiv A F_{p-1} \bmod p$ with $A, B \in \mathbb{F}_{p}$. Therefore for any $\beta \in \mathbb{Z}$

$$
(S+\beta J) \mid U^{R} \equiv(\beta A+B) F_{p-1} \bmod p .
$$

It follows from $(7)$ that $A \not \equiv 0 \bmod p$. Let $\xi(P) \equiv-B / A \bmod p$. If $\beta \not \equiv \xi(P) \bmod p$ then $\beta A+B \not \equiv 0 \bmod p$ and (8) follows from this observation, (9), and (6). 


\section{References}

[1] Shigeki Akiyama, On the $2^{n}$ divisibility of the Fourier coefficients of $J_{q}$ functions and the Atkin conjecture for $p=2$, Analytic number theory and related topics (Tokyo, 1991), 1-15, World Sci. Publishing, River Edge, NJ, 1993.

[2] A. O. L. Atkin, Congruences for modular forms, 1968 Computers in Mathematical Research pp. 8-19 North-Holland, Amsterdam.

[3] Congruence Hecke operators, Number Theory, Proc. Sympos. Pure Math. 12, 33-40, (1969).

[4] A. O. L. Atkin and J. N. O'Brien, Some properties of $p(n)$ and $c(n)$ modulo powers of 13, Trans. Amer. Math. Soc. 126 (1967) 442-459.

[5] Jan H. Bruinier and Ken Ono, The arithmetic of Borcherds' exponents, Math. Ann. 327 (2003), no. 2, 293-303.

[6] Bernard Dwork, The $U_{p}$ operator of Atkin on modular functions of level 2 with growth conditions, Modular functions of one variable, III (Proc. Internat. Summer School, Univ. Antwerp, Antwerp, 1972), pp. 57-67. Lecture Notes in Math., Vol. 350, Springer, Berlin, 1973.

[7] Haruzo Hida, Elementary theory of L-functions and Eisenstein series, London Mathematical Society Student Texts, 26, Cambridge University Press, Cambridge, 1993.

[8] Masanobu Kaneko and Don Zagier, Supersingular j-invariants, hypergeometric series, and Atkin's orthogonal polynomials, Computational perspectives on number theory (Chicago, IL, 1995), 97-126, AMS/IP Stud. Adv. Math., 7, Amer. Math. Soc., Providence, RI, 1998.

[9] Nicholas M. Katz, p-adic properties of modular schemes and modular forms, Modular functions of one variable, III (Proc. Internat. Summer School, Univ. Antwerp, Antwerp, 1972), pp. 69-190. Lecture Notes in Mathematics, Vol. 350, Springer, Berlin, 1973.

[10] Masao Koike, Congruences between modular forms and functions and applications to the conjecture of Atkin, J. Fac. Sci. Univ. Tokyo Sect. IA Math. 20 (1973), 129-169.

[11] Morris Newman, Congruences for the coefficients of modular forms and for the coefficients of $j(\tau)$, Proc. Amer. Math. Soc. 91958 609-612.

[12] Jean-Pierre Serre, Formes modulaires et fonctions zêta p-adiques, Modular functions of one variable, III, Proc. Internat. Summer School, Univ. Antwerp, 1972, pp. 191-268, Lecture Notes in Math., Vol. 350, Springer, Berlin, 1973.

[13] _ Divisibilité de certaines fonctions arithmétiques, Enseignement Math. (2) 22 (1976), no. 3-4, 227-260.

Department of Mathematics, Temple University, 1805 N. Broad St., Philadelphia, PA 19122

E-mail address: pasha@temple.edu 